\title{
Effect of environment on the tribological behavior of Si-incorporated diamond-like carbon films
}

\author{
Seung Ho Yang ${ }^{a}$, Hosung Kong ${ }^{a}, *$, Kwang-Ryeol Lee ${ }^{b}$, Sejun Park ${ }^{b}$, Dae Eun Kim ${ }^{c}$ \\ a Tribology Research Center, Korea Institute of Science and Technology, Seoul, South Korea \\ ${ }^{\mathrm{b}}$ Thin Film Technology Research Center, Korea Institute of Science and Technology, Seoul, South Korea \\ ${ }^{c}$ Department of Mechanical Engineering, Yonsei University, Seoul, South Korea
}

Received 21 February 2001; received in revised form 15 August 2001; accepted 26 September 2001

\begin{abstract}
An experimental study was performed to discover the effect of environment on the tribological behavior of Si-incorporated diamond-like

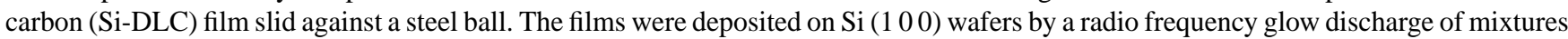
of benzene and dilute silane gases. Experiments using a ball-on-disk test-rig were performed in vacuum, dry air and ambient air. It was observed that coefficient of friction decreased as the environment changed from vacuum to dry air. Results also showed that low and stable friction related closely to the smoothening of track surfaces and the formation of silicon-rich oxide debris. (C) 2002 Elsevier Science B.V. All rights reserved.
\end{abstract}

Keywords: Diamond-like carbon film; Plasma-assisted chemical vapor deposition; Silicon; Wear debris; Environment

\section{Introduction}

Diamond-like carbon (DLC) film has been one of the hot research topics in the field of engineering [1]. Because of its superior tribological characteristics such as low coefficient of friction and high wear resistance, DLC film has been applied to the contacting surfaces of machine elements and magnetic storage devices. Bearing elements of spacecrafts, disk and slider surfaces of hard disk drive (HDD) are such examples, all of which require low and stable friction, low wear rate, and extreme reliability in a wide range of environment [2].

Generally, DLC films show coefficients of friction in the range of $0.1-0.3$ that correspond to the values of typical solid lubricants. Some researchers showed that, by changing the microstructure or chemical composition of DLC films, it is possible to obtain much lower friction. Multilayer DLC films [3], functionally gradient coatings [4], and nanocomposite coatings [5] are the examples. Recently, attempts have also been made to decrease the coefficient of friction by adding a third element. Silicon-incorporated DLC (Si-DLC) film is one of them [6-10]. Oguri and Arai [6,8] reported a very low coefficient of friction of Si-DLC film. Kim et al. [7] also reported the coefficient of friction less than 0.1 in ambient air.

\footnotetext{
* Corresponding author. Present address: P.O. Box 131, Cheongryang, Seoul 130-650, South Korea, Tel.:+82-2-958-5664; fax: +82-2-958-5659. E-mail address: hkong@kist.re.kr (H. Kong).
}

It is known that the tribological behavior of DLC films strongly depends on the environmental conditions. Miyoshi et al. [4] recently studied the friction and wear behavior of functionally graded DLC films in humid air and ultra-high vacuum. He showed that humid air provides the films with preferable tribological performances in terms of coefficient of friction and wear rate, while the investigated films exhibited severe wear in ultra-high vacuum. Many other researchers $[2,6,8]$ also argued that the tribological behavior of DLC films is significantly influenced by the test environment.

The aim of the present study is to clarify the effect of environment on the tribological characteristics of Si-DLC film. Frictional behaviors of Si-DLC films slid against a steel ball were investigated in vacuum, ambient air and dry air. In this work, we focused on the role of wear debris and proposed the mechanism for low friction.

\section{Experimental details}

\subsection{Si-DLC deposition}

Si-DLC films were deposited on p-type Si (1 00$)$ wafers by a radio frequency plasma-assisted chemical vapor deposition (RF PACVD) method. Details of the deposition were described elsewhere [11]. The films were deposited at the bias voltage of $400 \mathrm{~V}$ and deposition pressure of 
1.33 Pa. Mixtures of benzen $\left(\mathrm{C}_{6} \mathrm{H}_{6}\right)$ and dilute silane $\left(\mathrm{SiH}_{4}+\right.$ $\mathrm{H}_{2}$ ) were used for the deposition. The deposition time was adjusted to obtain $1 \mu \mathrm{m}$ thick film in all diluted silane fractions. The composition of the film was measured by Rutherford backscattering spectroscopy (RBS) and elastic recoil detection (ERD) analysis using a collimated ${ }^{4} \mathrm{He}^{2+}$ beam of $2.2 \mathrm{MeV}$. The silicon and hydrogen concentrations as a function of dilute silane are presented in Fig. 1. The silicon concentration in the films gradually increased to 2 at. $\%$ with increasing amount of dilute silane in the mixture up to $60 \%$. Further increasing the fraction of dilute silane significantly increased the silicon concentration to 9.5 at. \%, when the dilute silane fraction was $90 \%$. Total hydrogen concentrations decreased slightly from 18 to $15 \%$ with increasing dilute silane fraction. Deposited film surfaces were very smooth; the root mean square roughness of the surface measured by an atomic force microscope was less than $10 \mathrm{~nm}$. Film thickness was measured by an Alpha-step (Tencor P-1).

\subsection{Tribo-tester and the test conditions}

Friction and wear were measured with a ball-on-disk tribo-tester. The overall view of the equipment is presented

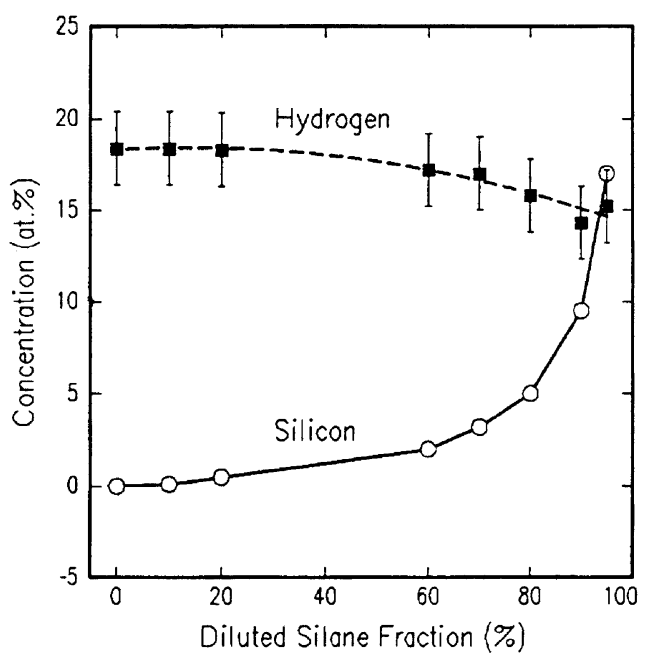

Fig. 1. Composition of Si-DLC films for various silane fractions.

in Fig. 2. The apparatus was described in detail elsewhere [12]. Tests were performed in vacuum $\left(8 \times 10^{-3} \mathrm{~Pa}\right)$, ambient air and dry air (less than 5\% relative humidity). Relative humidity in the test chamber was measured by a digital

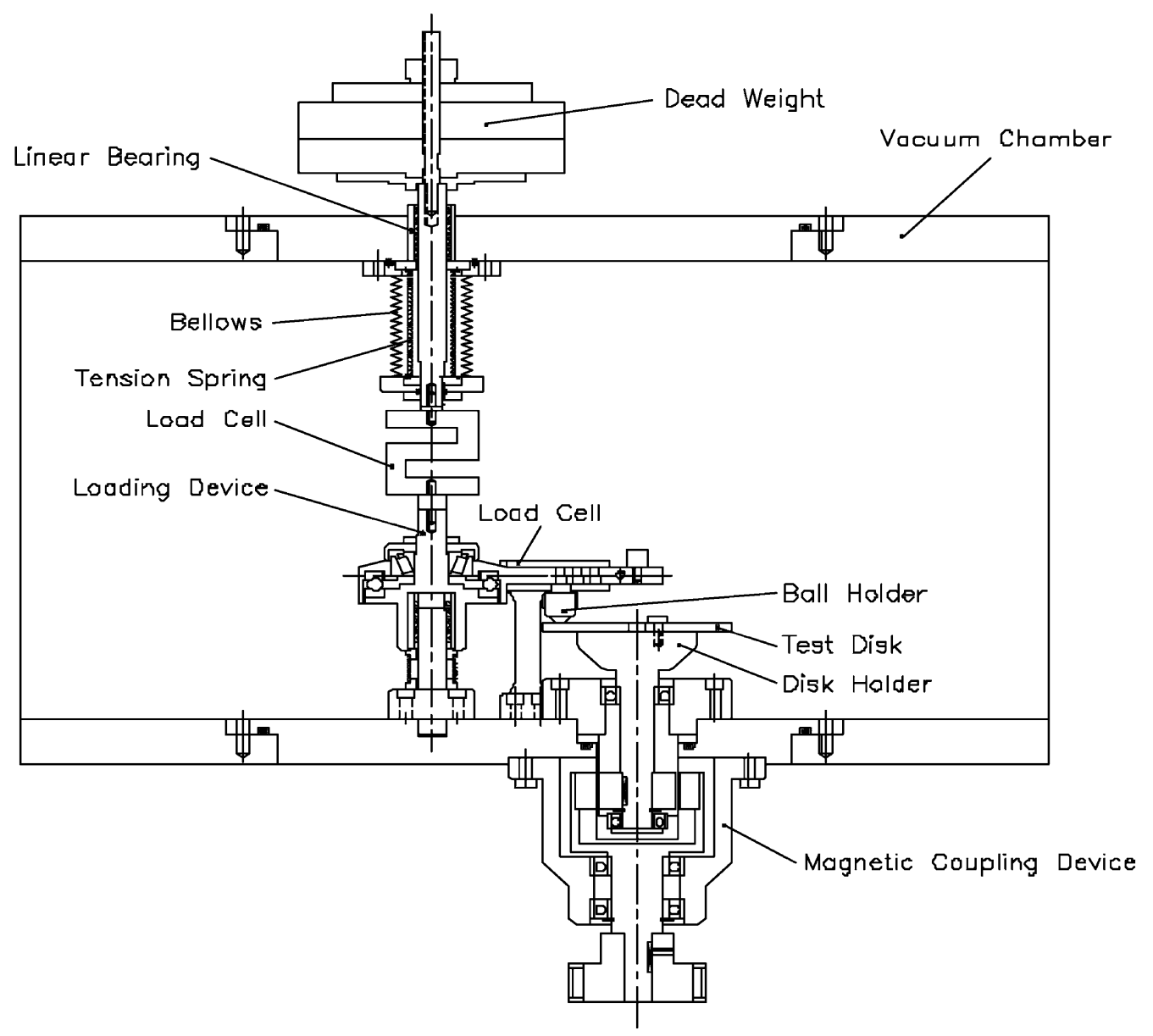

Fig. 2. A schematic view of the experimental setup. 
Table 1

Deposition conditions for Si-DLC thin film

\begin{tabular}{|c|c|}
\hline Method & Radio frequency PACVD \\
\hline Processing gas & $\mathrm{C}_{6} \mathrm{H}_{6}+$ diluted $\mathrm{SiH}_{4}$ \\
\hline Deposition pressure & $1.33 \mathrm{~Pa}$ \\
\hline Bias voltage & $-400 \mathrm{Vb}$ \\
\hline Diluted silane fraction & $0-90 \%$ \\
\hline Substrate & p-type $\left(\begin{array}{lll}1 & 0 & 0\end{array}\right) \mathrm{Si}$ wafer \\
\hline
\end{tabular}

hygrometer. In the dry air condition, test chamber was first evacuated and then dry air was charged and maintained throughout the test. The specimens were bonded on a steel disk using a double-side adhesive tape. Steel ball (AISI $52100,12.7 \mathrm{~mm}$ diameter) slid against the surface. All of the tests were performed in unlubricated conditions at the speed of $100 \mathrm{~mm} / \mathrm{sec}$ for a fixed number of cycles $(5104$, sliding distance: $7854 \mathrm{~m}$ ). The normal load was $9.8 \mathrm{~N}$. After the tests, wear tracks and debris were analyzed by an optical microscope, scanning electron microscope (SEM), Auger electron spectroscopy (AES) and atomic force microscope (AFM). Detailed test conditions are listed in Tables 1 and 2. Friction torque was measured with a strain-gauge type load cell. In this work, the coefficient of friction was defined as the ratio of measured tangential force divided by

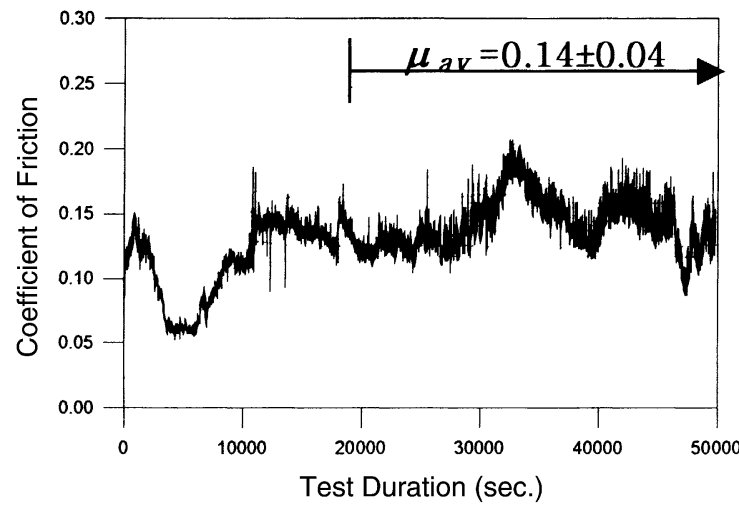

(a) 0 at $\% \mathrm{Si}$

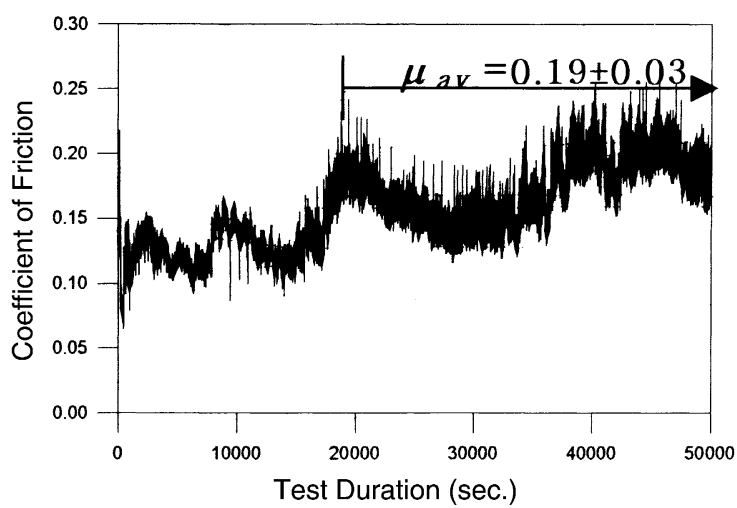

(c) 2 at $\% \mathrm{Si}$
Table 2

Test conditions

\begin{tabular}{lll}
\hline Test conditions & \multicolumn{2}{l}{ Specimen } \\
\cline { 2 - 3 } & Ball & Si wafer \\
\hline Roughness, Ra (nm) & 10 & 3 \\
Coating thickness $(\mathrm{nm})$ & - & 1000 \\
Speed $(\mathrm{mm} / \mathrm{s})$ & 100 & \\
Si incorporated (at.\%) & $0,0.5,2,9.5$ \\
Normal force (N) & 9.8 (Hertzian maximum \\
& pressure; 832 MPa) \\
Lubrication & Dry \\
Environments & Ambient air, dry air, vac- \\
Temperature & uum $\left(8 \times 10^{-3} \mathrm{~Pa}\right)$ \\
& Room temperature
\end{tabular}

the normal force. Each test was repeated more than three times.

\section{Results and discussion}

\subsection{Results in vacuum, ambient air and dry air}

Fig. 3 shows the test results in vacuum. In this test, vacuum $\left(8 \times 10^{-3} \mathrm{~Pa}\right)$ was maintained throughout the test. High

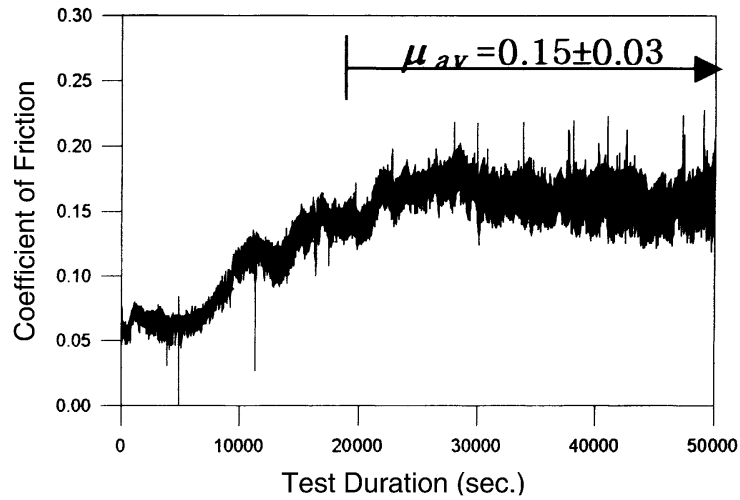

(b) 0.5 at\% $\mathrm{Si}$

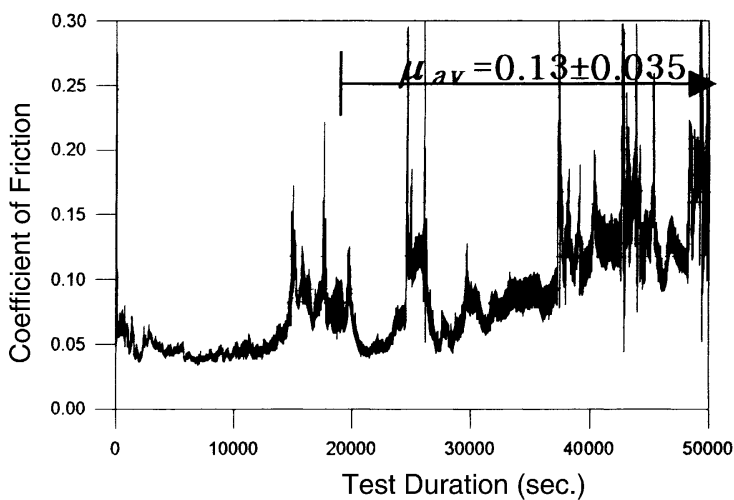

(d) 9.5 at\% Si

Fig. 3. Frictional behavior in vacuum $\left(8 \times 10^{-3} \mathrm{~Pa}\right)$. 
and unstable friction was observed in vacuum. The effect of $\mathrm{Si}$ incorporation on the coefficient of friction was not clear: average coefficient of friction increased with the Si content by 2 at. $\%$, but it dropped down and revealed similar value to that of pure DLC films for the 9.5 at.\% Si-DLC film. Throughout this work, the average coefficient of friction was taken from 20,000 to 50,000 contact cycles where it was relatively stable.

Results in ambient air are presented in Fig. 4 (a). In this test, relative humidity ranged from 40 to $50 \%$. In ambient air, the coefficients of friction were observed to be lower and much more stable than those in vacuum. Fig. 4 (b) shows
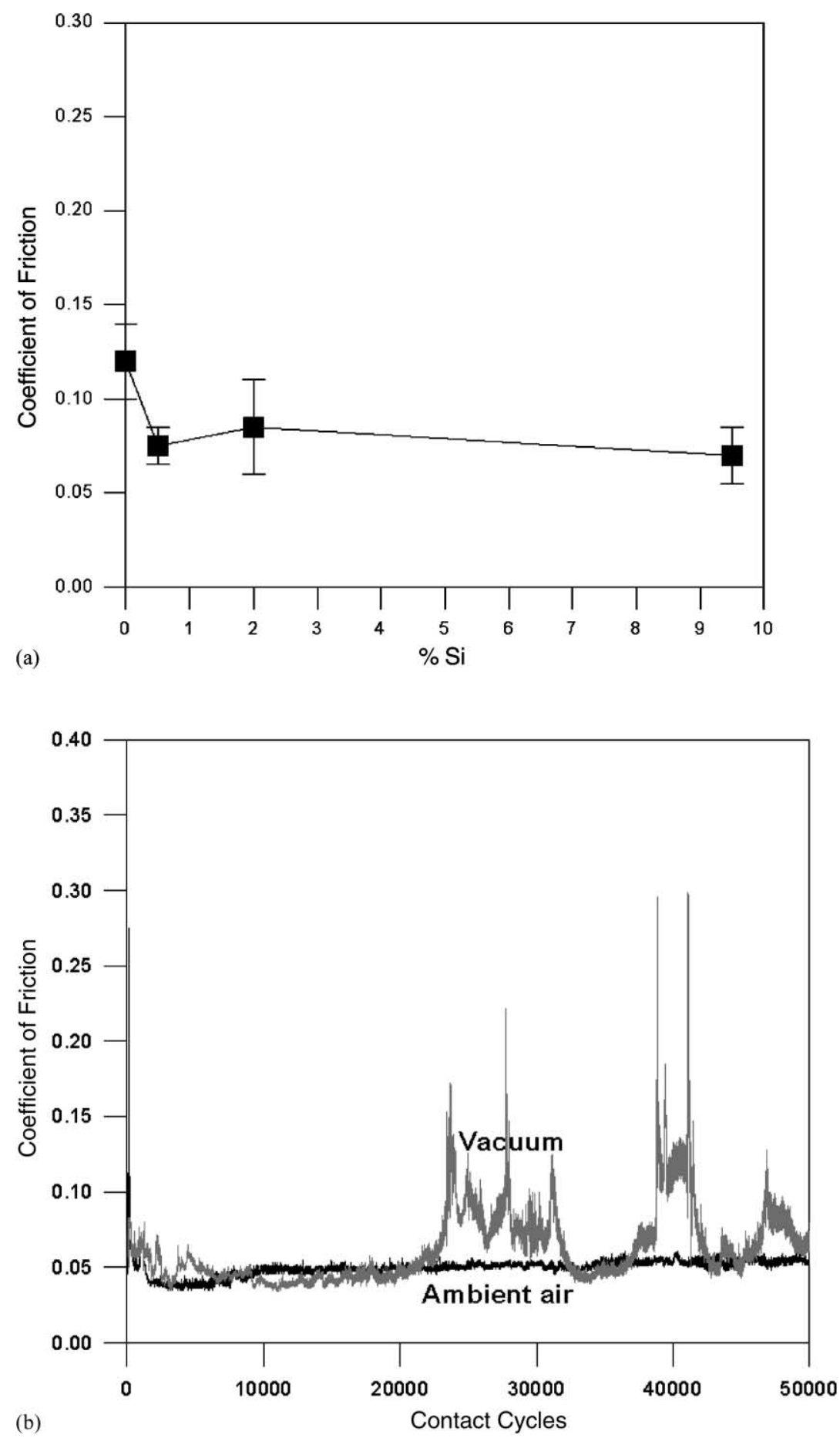

Fig. 4. Frictional behavior in ambient air (RH 40-50\%) (a) average coefficient of friction vs. Si\% in ambient air and (b) frictional behavior of 9.8 at. $\%$ Si-DLC in vacuum and ambient air. 


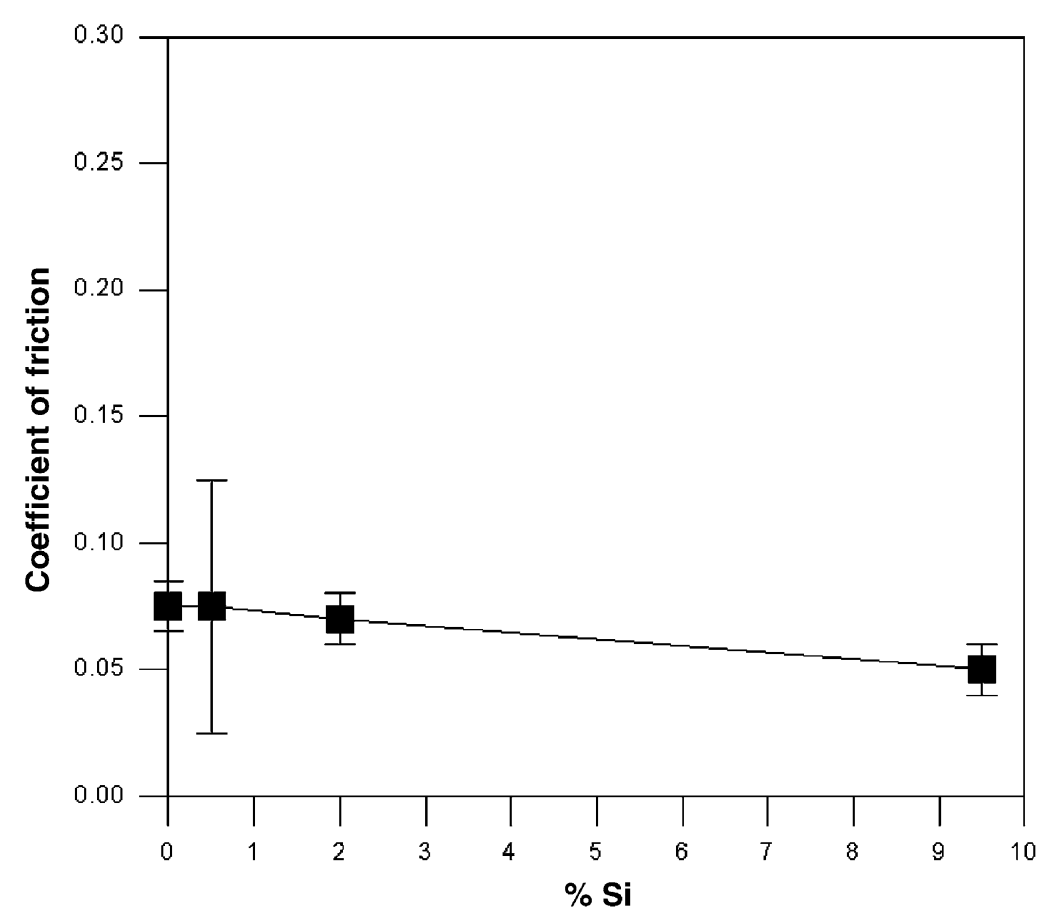

Fig. 5. Average coefficient of frictional vs. Si\% in dry air.

a typical example of the 9.5 at.\% Si-DLC film compared to the same film in vacuum. The average coefficient of friction was very low at about 0.06 . It was also found that the coefficients of friction of DLC films decreased with increasing Si content.

Fig. 5 shows the average coefficients of friction measured in dry air. Results showed that the friction was the most stable and lowest among the test conditions: the coefficient of friction ranged from 0.04 to 0.06 . However, the silicon content in DLC film did not have any significant influence on the frictional behavior. These results showed clearly that frictional properties of Si-DLC films are influenced by the changes in environmental conditions.

\subsection{Discussion}

In Fig. 6 average coefficients of friction are summarized as a function of test environment. Results showed that the average coefficient of friction was decreased notably when the environment is changed from vacuum to dry air. In ambient air, the coefficient of friction was slightly higher than that in dry air.

Wear characteristics of ball and DLC-coated surfaces are summarized in Figs. 7 and 8, respectively. In this work, the linear wear rate was defined as the worn depth divided by the sliding distance. Both results also showed that the wear characteristics were greatly affected by environment. For balls, the linear wear rate was the lowest in ambient air. On the other hand, DLC films showed the lowest linear wear rate in vacuum. This was interesting since average coefficient of friction was the highest in vacuum. The effect of $\mathrm{Si}$ incorporation on the wear was not found obvious in each environmental condition.

Micrographs of balls and DLC films are presented in Figs. 9 and 10, respectively. In these results, 2 at.\% Si-DLC coated specimens were selected because they showed the largest difference in the coefficient of friction with respect

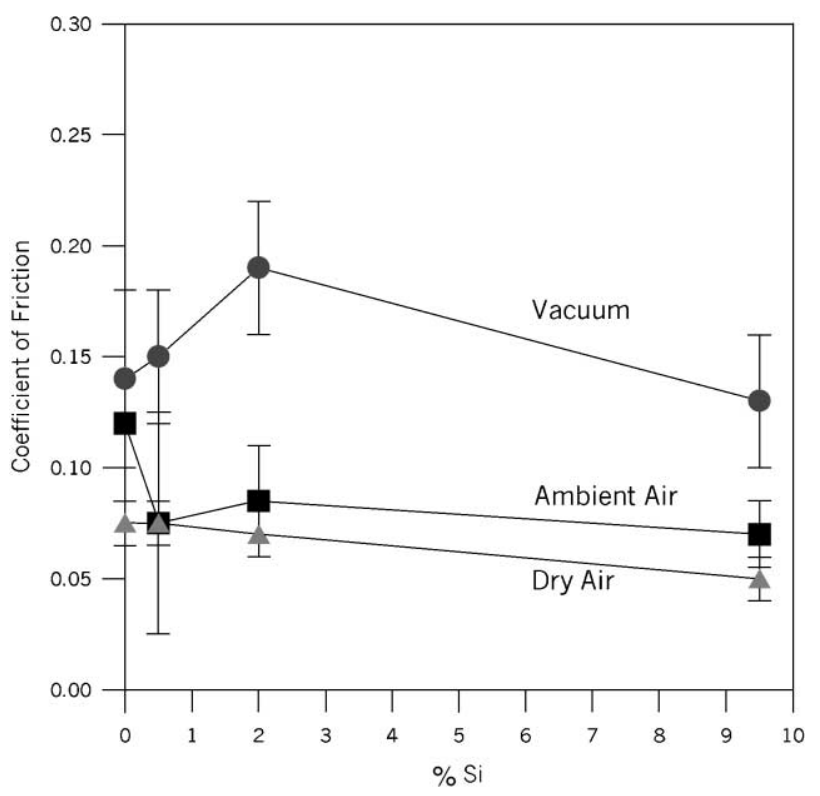

Fig. 6. Average coefficients of friction in vacuum, ambient air and dry air 


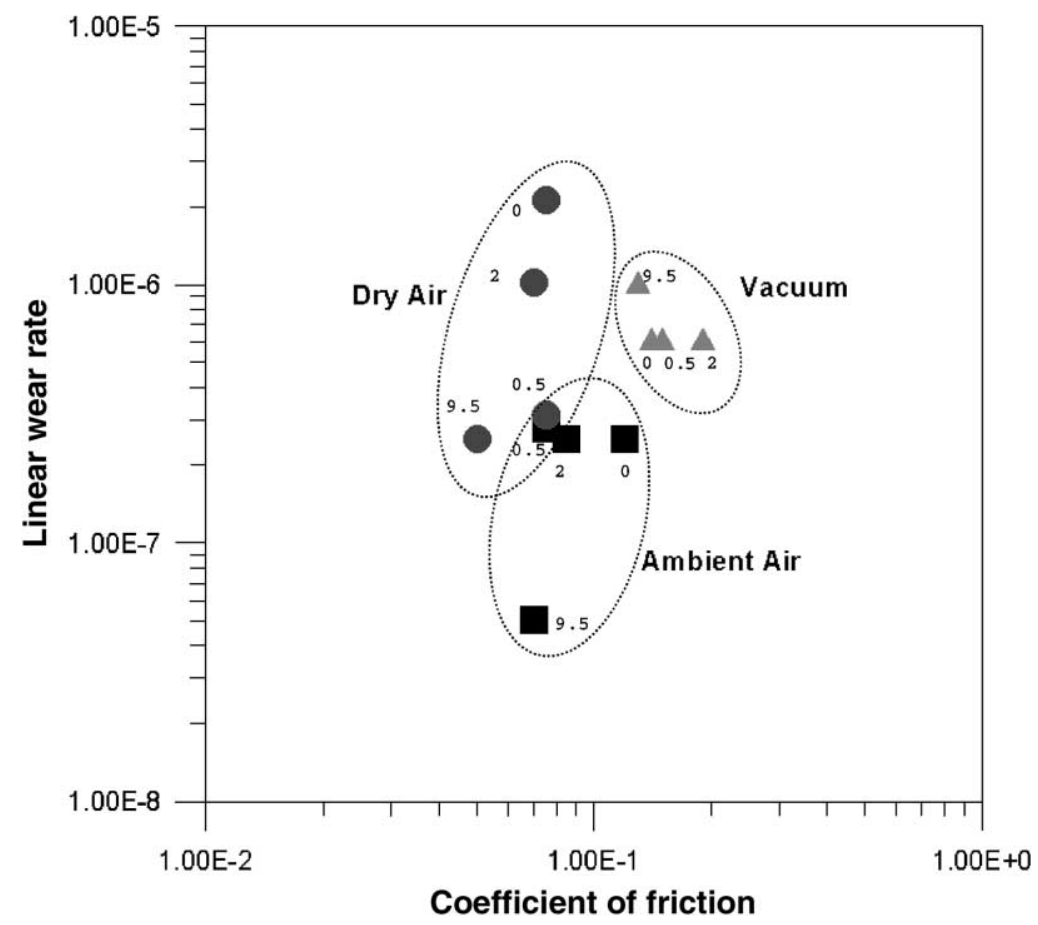

Fig. 7. Variations of the linear wear rate of balls in various environments (arabic number represents each at.\% of silicon).

to the environment as shown in Fig. 6. It could be noted that worn surfaces revealed characteristic features associated with each wear mechanism with respect to the environmental condition. In vacuum, transferred films and wear debris were hardly observed on the ball scar. There were evidences that wear debris plowed the DLC film and generated transferred layers on the wear track (Fig. 10a). On the other hand, ball scar in ambient air was largely covered by the

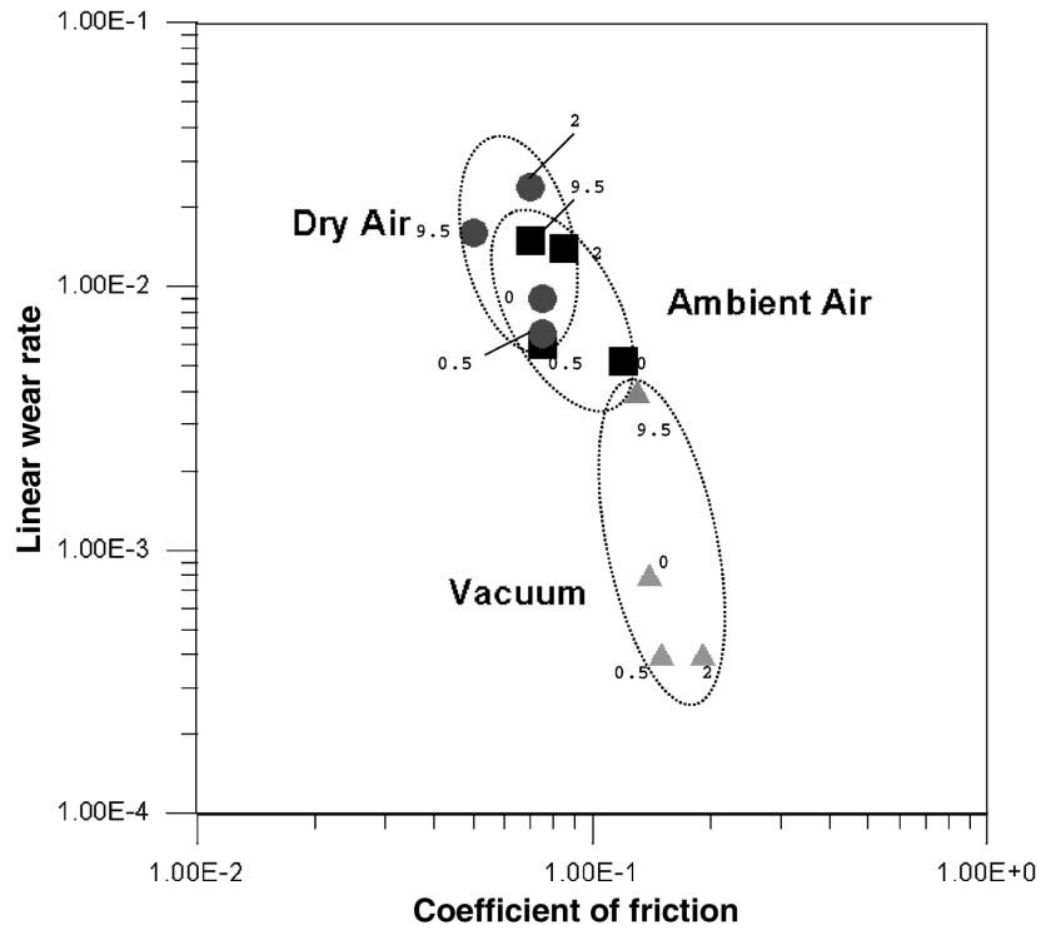

Fig. 8. Variations of the linear wear rate of tracks in various environments (arabic number represents each at.\% of silicon). 

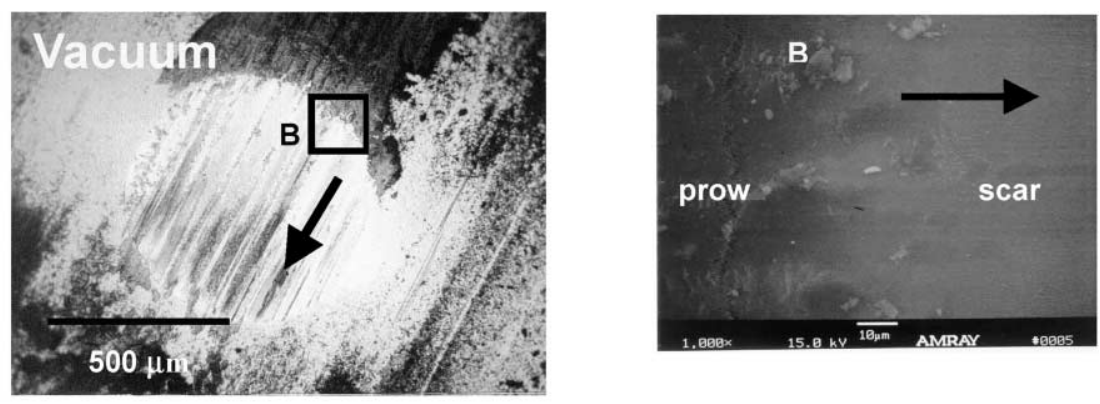

(a) vacuum
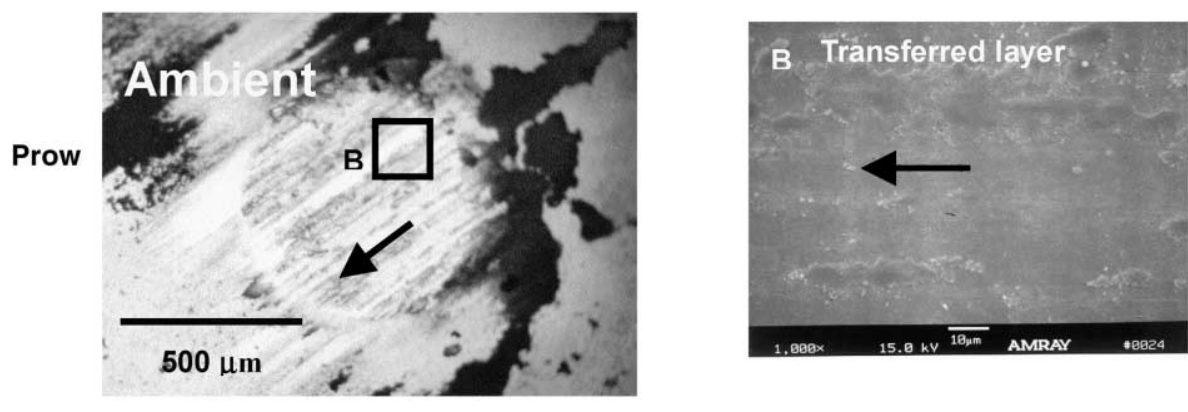

(b) ambient air
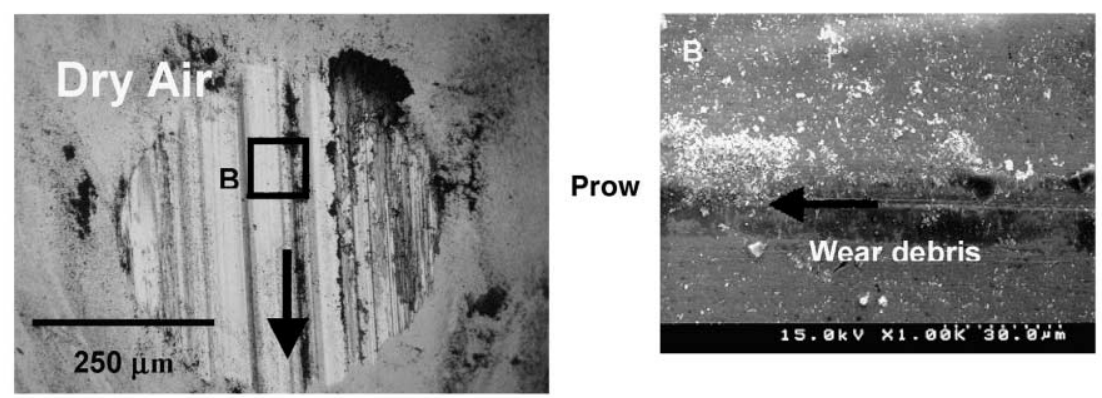

(c) dry air

Fig. 9. Micrographs of tested ball surfaces (the sliding direction is shown by black arrows).

transferred layers that were formed by agglomerated small debris (Fig. 9b). It seemed that this transferred film acted as a protective layer and it depressed the wear of the ball. Wear track in ambient air also showed narrow center-line strips (Fig. 10b) which were formed by the agglomerated debris. Also, scratch was not observed on the surfaces. Ball surfaces in dry air showed many strip lines parallel to the sliding direction (Fig. 9c). SEM analysis showed that ground wear particles of small size (sub-micron) were scattered around the wear track and created strip-like patterns without agglomeration (Fig. 10c). Evidence of material transfer was hardly observed on the wear track. This suggested that the ground wear debris have a weak adhesion with the wear track. It could be attributed to a weak capillary force due to little condensed water. It seems that the ground wear debris of small size was likely to roll easily [13].
This may explain why the friction was low and stable in dry air.

Wear tracks were also analyzed by an atomic force microscope as presented in Fig. 11. It could be found that many scratch patterns were observed on the track in vacuum. On the other hand, wear track in ambient air showed smooth humps. Wear track in dry air also revealed smooth surface, which were ground by small wear debris (Fig. 10c).

Wear debris on the ball scar and wear track were chemically analyzed by Auger spectroscopy, which is presented in Fig. 12. For this analysis, 9.5 at.\% Si-DLC specimens were selected. In order to compare the results between the samples, the spectra were normalized with respect to the oxygen signal of unity. In Fig. 12 it is worth noting that in vacuum the carbon content of the debris is very small but appears much exaggerated during the normalization. By changing the 

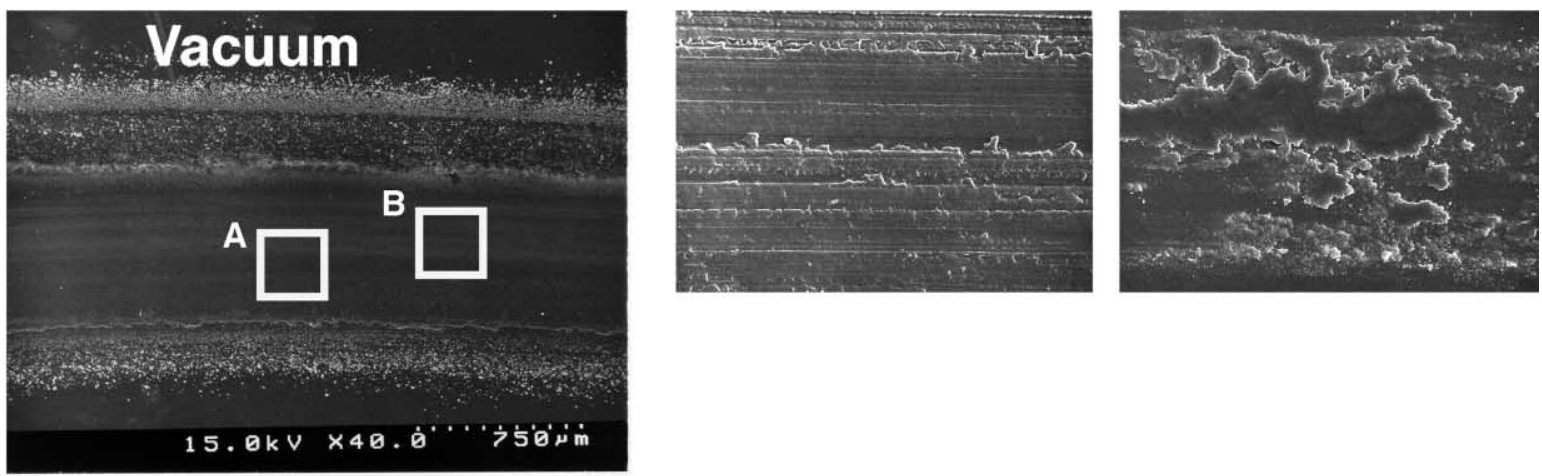

(a) vacuum
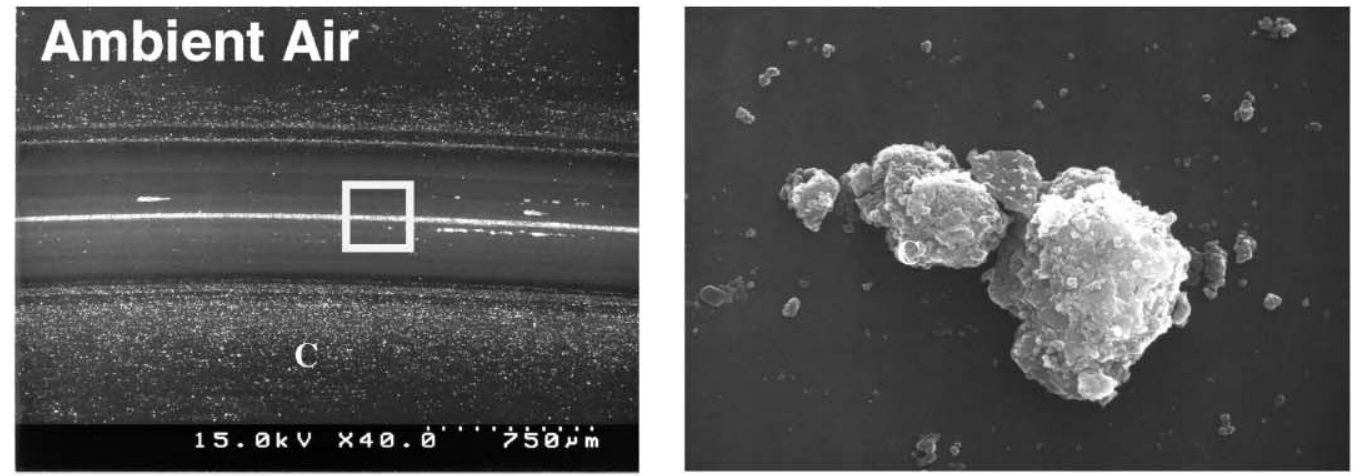

(b) ambient air
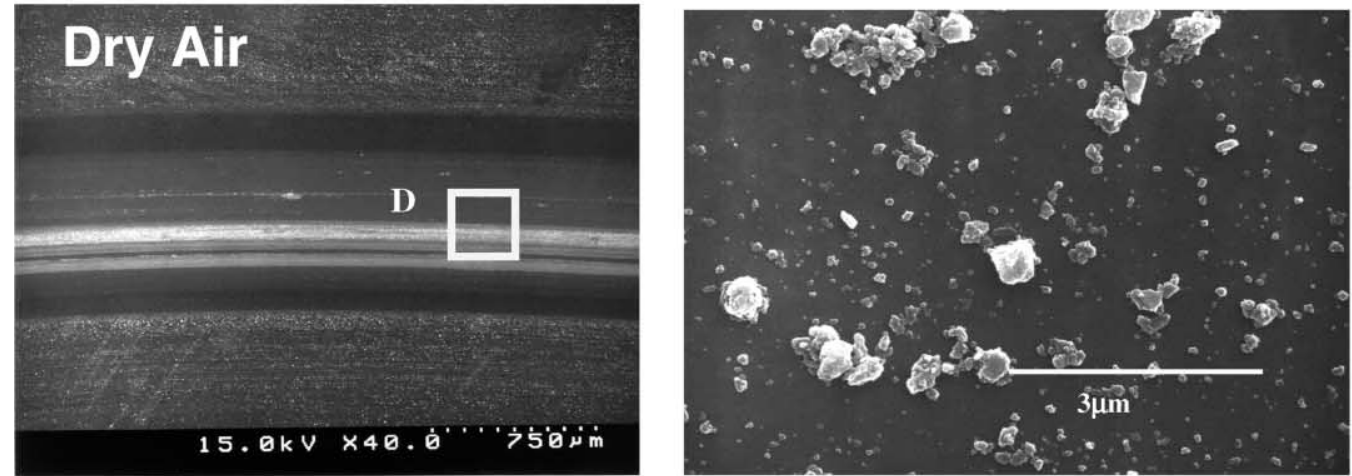

(c) dry air

Fig. 10. Micrographs of tested Si-DLC track surfaces.

environment from vacuum to dry air, the iron content of the debris decreased while the silicon content increased. Therefore, it was found that the debris covered on the ball scar and track in dry air was not ferrous oxide but silicon-rich oxide.

Test results described above revealed that friction occurred in various mechanisms with respect to the test environments. We, therefore, propose each friction mechanism as follows. In vacuum, it was plowing of the wear debris and shearing at the transferred layers that governed the frictional behavior and caused the high fluctuation in friction.
In ambient air, formation of the transferred layer governed the friction behavior, which protected the ball surface. Also, the life cycle of the transferred layer was believed to be a cause of the fluctuation in friction. In ambient air, plowing was neglected since the scratch patterns were not observed on the ball and DLC surfaces (Figs. 9 and 10). In dry air, the most favorable feature in the friction mechanism was the lubricating effect caused by ground fine wear debris of silicon-rich oxide. As a result, low and stable friction was sustained in dry air. 
(a) vacuum

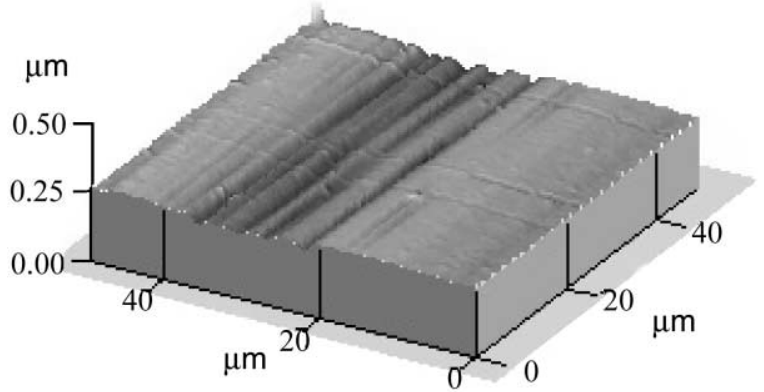

(b) ambient air

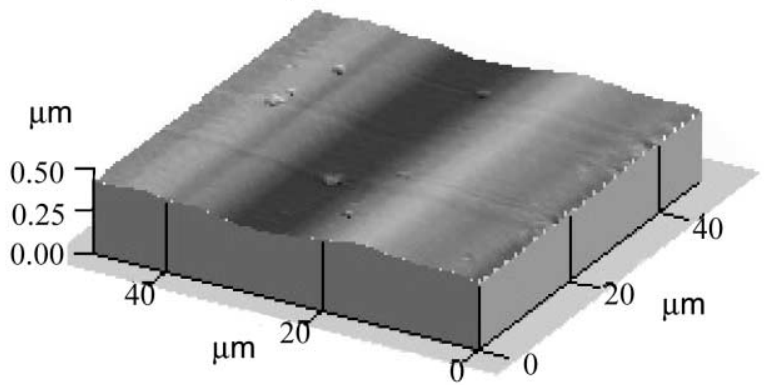

(c) dry air

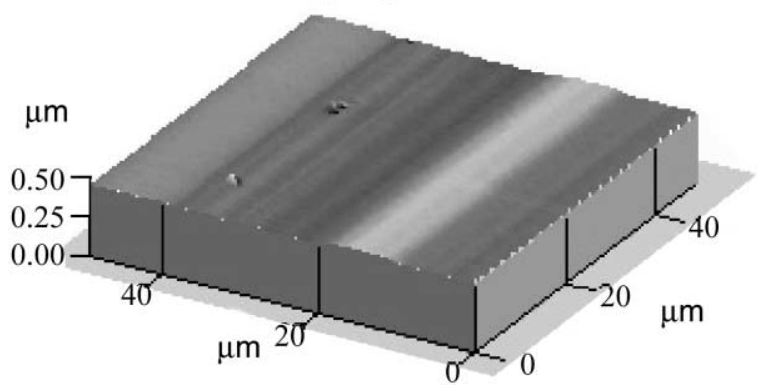

Fig. 11. Atomic force microscope (AFM) surface images of 2 at.\% Si-DLC track surfaces.

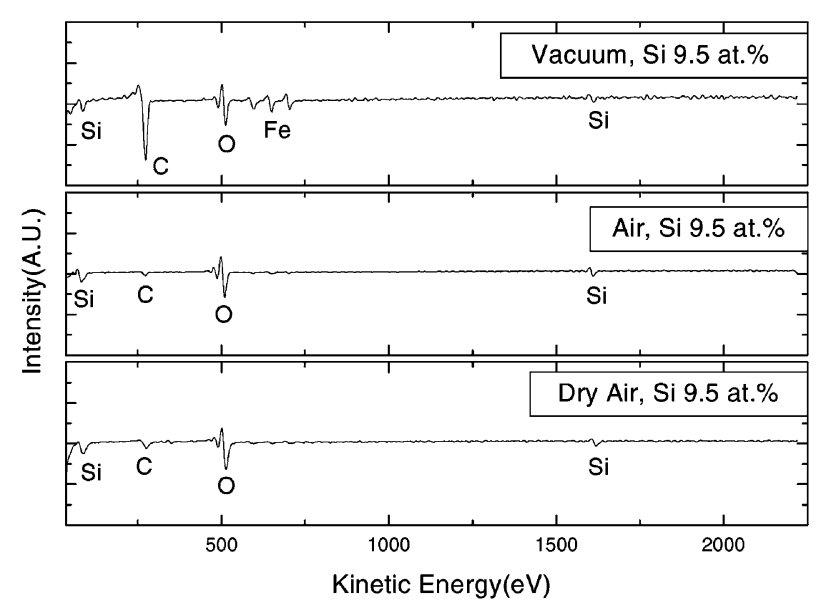

(a) ball surfaces

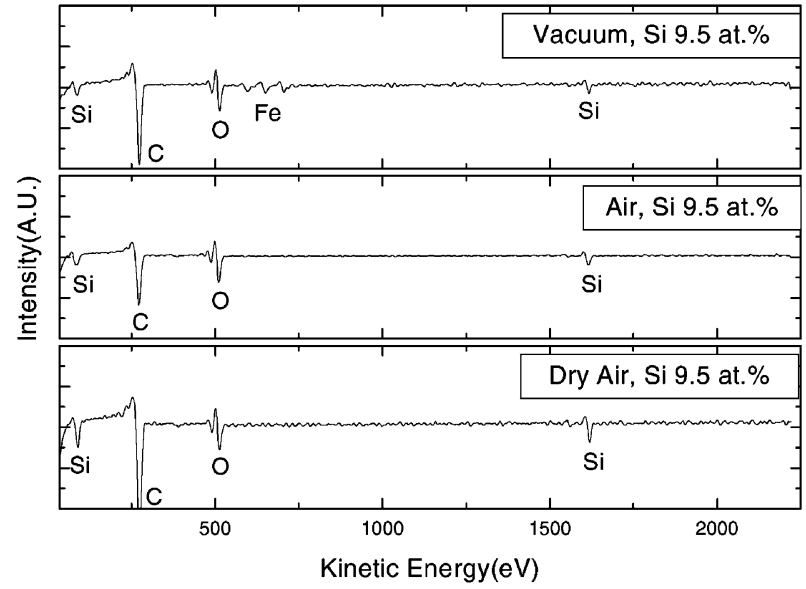

(b) track surfaces

Fig. 12. Auger spectra of the wear debris on the ball and track surfaces.

\section{Conclusions}

The experimental results of this work can be summarized as follows:

1. The tribological behavior of Si-diamond-like carbon film was strongly governed by the test environment.

2. Coefficient of friction of the film decreased significantly as the environment changed from vacuum to dry air.

3. The tribological behavior of Si-DLC films was greatly affected by the formation of mass transferred layer.
4. Low and stable frictional behavior in dry air was attributed to the smoothening of track surface. Also, the small size debris of silicon-rich oxide was closely related to the low and stable friction in dry air.

\section{Acknowledgements}

The authors would like to thank the Korea Ministry of Science and Technology and Critical Technology 21 program 
(Machinery Design Technology Enhancement) and National Laboratory Program for their support and interest in this work.

\section{References}

[1] B. Bhushan, Chemical, mechanical and tribological characterization of ultra-thin and hard amorphous carbon coatings as thin as $3.5 \mathrm{~nm}$ recent developments, Diamond Relat. Mater. 8 (11) (1999) 19852015.

[2] C. Donnet, M. Belin, J.C. Auge, J.M. Martin, A. Grill, V. Patel, Tribochemistry of diamond-like carbon coatings in various environment, Surf. Coat. Technol. 68/69 (1994) 626-631.

[3] A.A. Voevodin, J.M. Schneider, C. Rebholz, A. Matthews, Multilayer composite ceramic-metal-DLC coatings for sliding wear applications, Tribol. Int. 29 (7) (1996) 559-570.

[4] K. Miyoshi, B. Pohlchuck, K.W. Street, J.S. Zabinski, J.H. Sanders, A.A. Voevodin, R.L.C. Wu, Sliding wear and fretting wear of diamond-like carbon-based, functionally graded nanocomposite coatings, Wear 225-229 (Part 1) (1999) 65-73.

[5] A.A. Voevodin, J.P. O'Neill, J.S. Zabinski, Nanocomposite tribological coatings for aerospace applications, Surf. Coat. Technol. $116 / 119$ (1999) 36-45.
[6] K. Oguri, T. Arai, Low friction coatings of diamond-like carbon with silicon prepared by plasma assisted chemical vapor deposition, J. Mater. Res. 5 (11) (1991) 259-268.

[7] M-G. Kim, K-R. Lee, K.Y. Eun, Tribological behavior of silicon-incorporated diamond-like carbon films, Surf. Coat. Technol. 112 (1999) 204-209.

[8] K. Oguri, T. Arai, Tribological properties and characterization of diamond-like carbon coatings with silicon prepared by plasmaassisted chemical vapor deposition, Surf. Coat. Technol. 47 (1991) $710-721$.

[9] J.F. Zhao, P. Lemoine, Z.H. Liu, J.P. Quinn, P. Maguire, J.A. McLaughlin, A study of microstructure and nanomechanical properties of silicon-incorporated DLC films deposited on silicon substrates, Diamond Relat. Mater. 10 (3-7) (2001) 1070-1075.

[10] J.C. Damasceno, S.S. Camargo, F.L. Freire, R. Carius, Deposition of Si-DLC films with high hardness low stress and high deposition rates, Surf. Coat. Technol. 133/134 (2000) 247-252.

[11] K.-R. Lee, M.-G. Kim, S.-J. Cho, K.Y. Eun, T.Y. Seong, Structural dependence of mechanical properties of Si-incorporated DLC films deposited by RF PACVD, Thin Solid Films 308/309 (1997) 263-267.

[12] E.-S. Yoon, H. Kong, O.K. Kwon, J.-E. Oh, Evaluation of friction characteristics at a pin-on-disk apparatus with different dynamic parameters, Wear 203/204 (1997) 341-349.

[13] K. Holmberg, A concept for friction mechanisms of coated surfaces, Surf. Coat. Technol. 56 (1992) 1-10. 